Ann. Génét. Sél. anim., I980, 12 (3), 255-265.

\title{
Composantes génétiques de la croissance avant sevrage des races Angus, Hereford, Shorthorn et de leurs croisements avec la race Charolaise
}

\author{
Maria C. MIQUEL et Carlos LÓPEZ SAUBIDET \\ Instituto Nacional de Tecnologia Agropecuaria, \\ Estacion Experimental Regional Agropecuaria de Balcarce, \\ Balcarce (Provincia de Buenos-Aires), Argentina
}

\begin{abstract}
Résumé
Pour déterminer les effets de vigueur hybride et les différences dues aux composantes génétiques additives individuelles et maternelles entre différentes races, nous avons réalisé différentes combinaisons linéaires entre les estimées de moindres-carrés des effets des races et de leurs croisements, pour les caractères suivants : le poids à la naissance et au sevrage, le gain de poids journalier absolu et relatif de la naissance au sevrage. Les races concernées sont 1'AberdeenAngus, la Hereford, la Shorthorn et la Charolaise; outre les accouplements en race pure et les croisements réciproques entre les races d'origine britannique, les vaches de races britanniques ont aussi été accouplées avec des taureaux Charolais. 450 veaux nnés de 1960 à 1965 à 1 a Station Expérimentale de Balcarce (Argentine) ont été analysés. Les effets de vigueur hybride ainsi que les différences entre les composantes génétiques individuelles et maternelles des races britanniques, n'apparaissent pas statistiquement significatifs. La race Charolaise est supérieure aux races britanniques, respectivement en croisement et en race pure, pour le poids à la naissance (23 et $33 \mathrm{p}$. Ioo) et pour le poids au sevrage (I I et I 7 p. Ioo).
\end{abstract}

\section{Introduction}

L'objectif des croisements en races bovines à viande ne se limite pas seulement à l'exploitation de la vigueur hybride. En plus de la valeur génétique additive des races parentales concernées et de leurs effets de vigueur hybride, les croisements cherchent à utiliser la complémentarité entre les aptitudes paternelles d'une race et les qualités maternelles d'une autre race.

C'est dans ce contexte que depuis I959 à la " Estación Experimental Regional Agropecuaria " de 1'I.N.T.A. à Balcarce (Argentine), nous étudions divers croisements entre races bovines à viande. Les principaux résultats zootechniques concernant ces croisements ont été publiés antérieurement (LóPEZ SAUBIDET et al., 
I963; Departamento de Producción Animal, r974). Il ressort que la pratique du croisement entre ces races bovines permet l'obtention d'une croissance plus rapide, la production d'animaux moins gras et de carcasses de meilleure valeur bouchère.

Cette analyse a pour objectif, à partir des performances mesurées sur la croissance avant sevrage des veaux de ces divers types d'accouplements, d'évaluer l'importance relative des effets attribuables à 1'hétérosis et aux composantes génétiques additives individuelles et maternelles, entre les races $A$ berdeen-Angus, Hereford et Shorthorn. La connaissance de ces divers effets est nécessaire pour rechercher des plans d'accouplements en vue de maximiser l'exploitation commerciale des mères, ainsi que pour connaître l'origine génétique des différences entre races bovines. De plus dans cette étude, nous avons pu comparer les performances de la race Charolaise par rapport à celles des races britanniques, en croisement et en race pure.

\section{Matériel animal et méthodes d'analyse}

L'analyse a porté sur les performances de 450 veaux nés entre I 960 et I965 à la Station expérimentale 1'I.N.T.A. à Balcarce (Argentine); ces animaux font partie d'une expérience plus vaste de comparaison de différentes races et croisements pour la production de viande. L,es conditions de réalisation de cette expérience ont été décrites et critiquées par López SAUbidét et al. (r963) et le "Departamento de Production Animal " (r974).

Les veaux sont de race pure et croisés alors que les pères et les mères sont de race pure. Pour les 3 races d'origine britannique, Aberdeen-Angus, Hereford et Shorthorn, nous disposons des veaux nés des accouplements en race pure et des accouplements en croisement y compris les croisements réciproques. Pour la race Charolaise, seuls ont été réalisés les accouplements en race pure et ceux entre taureaux Charolais et vaches des trois races britanniques. En ce qui concerne les taureaux de races britanniques, ils appartenaient à un échantillon pris au hasard parmi les veaux avant la castration. Les taureaux (ou leur semence) Charolais étaient d'origine française. Tous les taureaux ou la semence de cet essai provenaient de reproducteurs pris au hasard parmi ceux dont la semence avait subi un test de fécondité.

L es veaux sont de race pure et croisés alors que les pères et les mères sont de race pure. Pour les 3 races d'origine britannique, Aberdeen-Angus, Hereford et Shorthorn. Les veaux ont été pesés à la naissance l'été de novembre à janvier ou février puis chaque 28 jours jusqu'au sevrage. Le gain de poids journalier absolu a été obtenu par régression du poids sur l'âge. Le gain du poids relatif ou croissance relative résulte de la régression du logarithme naturel du poids sur l'âge; ainsi, le gain de poids relatif exprime l'accroissement relatif du poids d'un animal par jour. Ce concept a été développé par TAYLOR (I97I) et discuté par KLOSTERMAN (I972). Le poids au sevrage a été ajusté à 1'âge de 2 ro jours par régression linéaire du poids sur l'âge (croissance absolue).

A partir de ces performances, nous avons fait une analyse de variance par moindres-carrés, selon un modèle incluant le type génétique du veau, son sexe, 1'année et le mois de naissance ainsi que l'âge de la mère lors de la naissance du veau. A partir de ces estimées de moindres-carrés et selon le modèle de décomposition des différentes composantes génétiques proposé par DrkERson (I969) et FOULLEY et LEFORT (I978), nous avons utilisé différentes combinaisons linéaires 
pour estimer les effets d'hétérosis individuels $\left(h^{\mathrm{I}}\right)$, les effets génétiques additifs directs $\left(g^{\mathbf{I}}\right)$ et maternels $\left(g^{\mathrm{M}}\right)$, ainsi que l'effet d'interaction (I) entre l'effet maternel moyen et le génotype du veau appelé aussi effet de "réciprocité ", pour les races britanniques. De même, une série d'autres combinaisons linéaires nous a permis de comparer la race Charolaise aux races britanniques, soit en race pure, soit en croisement sur vaches de races britanniques. Les tests d'hypothèse ont été réalisés en utilisant la méthodologie énoncée par HARVEY (I964); tous les tests ont été faits au niveau de probabilité de 0,05 .

\section{Résultats et discussion}

Dans le tableau I figurant pour chaque type génétique de veaux, le nombre d'observations, 1'estimée de moindres-carrés et 1'écart-type pour chacun des 4 caractères analysés.

\section{I. - Composantes génétiques des races britanniques}

Dans le tableau 2 sont rapportées les estimations des différentes composantes génétiques des caractères analysés obtenues à partir des estimées de moindrescarrés du tableau I. Seuls les effets génétiques directs $\left(g^{\mathrm{I}}\right)$ de la race Hereford pour le poids à la naissance sont statistiquement différents de zéro. Cependant, nous pouvons observer plusieurs tendances :

a) Effets génétiques additifs directs $\left(g^{\mathbf{I}}\right)$ : pour le poids à la naissance et au sevrage, ainsi que pour la croissance, les races se classent de la façon suivante : la Hereford est supérieure à 1'Angus, elle-même supérieure à la Shorthorn. Pour la croissance relative, 1'Angus est supérieure à la Hereford et à la Shorthorn.

b) Effets génétiques additifs maternels $\left(g^{\mathrm{M}}\right)$ : les mères Shorthorn tendent à avoir une incidence positive supérieure à celles des autres races sur tous les caractères de croissance avant sevrage, sauf pour la croissance relative où ce sont les mères Angus qui ont l'incidence la plus favorable.

c) Hétérosis sur les effets directs ou hétérosis individuel $\left(h^{\mathrm{I}}\right)$ : ces effets d'hétérosis direct varient entre -2 et $+5 \mathrm{p}$. Ioo, mais sont généralement inférieurs à 3 p. Ioo. Pour tous les caractères la combinaison Shorthorn $\times$ Hereford est celle qui tend à procurer les plus fortes valeurs d'hétérosis.

d) Effet de réciprocité $(I)$ : pour aucun des caractères analysés, l'interaction entre le génotype du veau et les effets maternels des mères n'atteint pas le niveau de la signification. Les valeurs sont plutôt faibles.

Les tendances observées dans le classement des races pour le poids à la naissance, la croissance absolue et le poids au sevrage, coïncident généralement avec celles rencontrées dans la littérature. Des trois races britanniques étudiées, la Hereford en général possède une valeur génétique supérieure pour les effets directs sur le poids de naissance et au sevrage ou la croissance absolue, suivie par 1'Aberdeen-Angus puis par la Shorthorn (GREGory et al., I965; GaINEs et al., I966; 


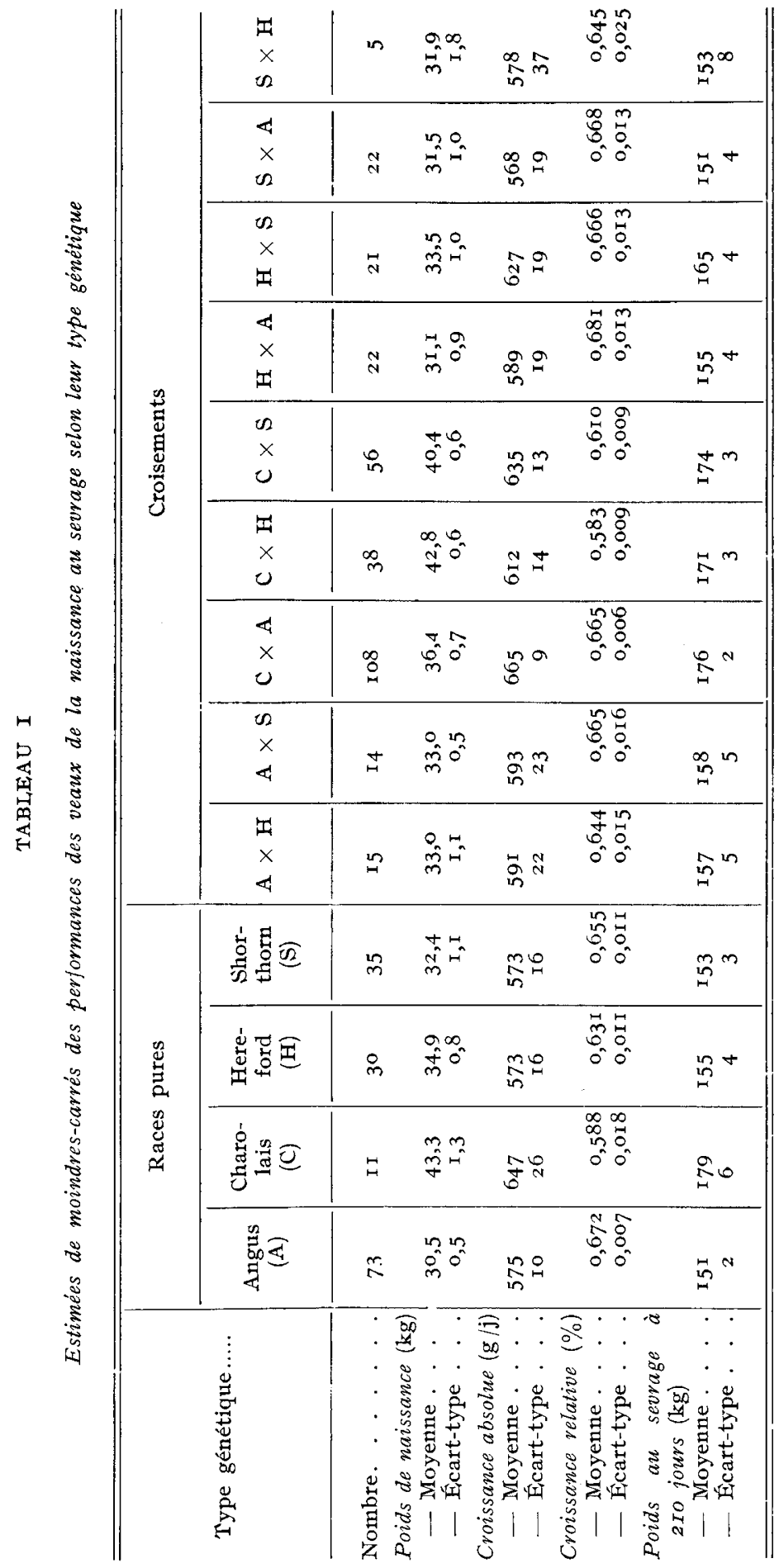


CROISSANCE, EN ANGUS, HEREFORD, SHORTHORN

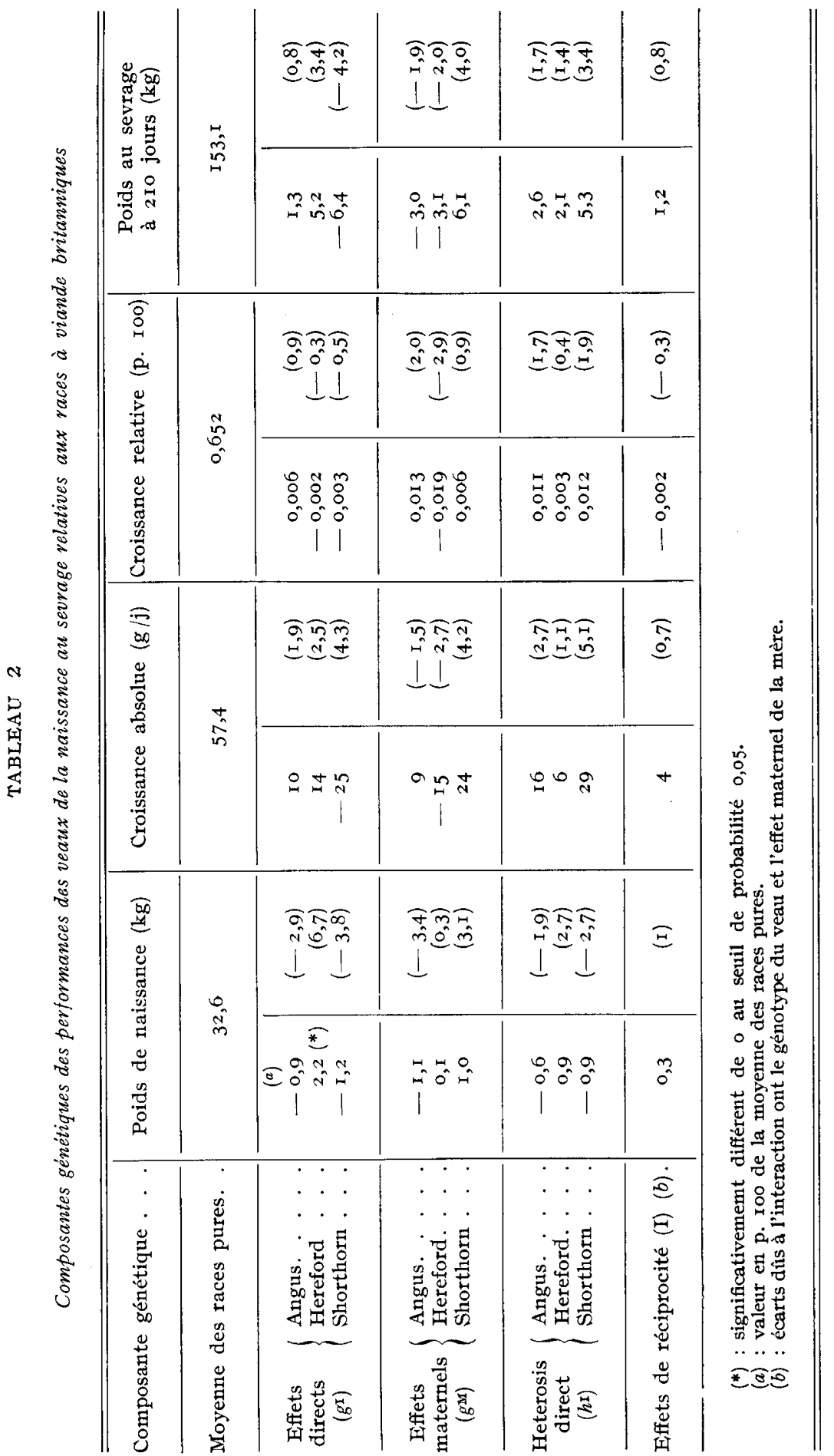


Cundiff, I970). Pour ces mêmes caractères, la valeur génétique de la race Shorthorn pour les effets maternels est, en général, supérieure à celle des deux autres races (GREGORY et al., I965; GAINEs et al., I966; CuNDIFF, I970). Les différences entre ces races trouvées dans la littérature sont également faibles, ce qui fait qu'elles ne sont pas toujours statistiquement significatives et que des différences de classement entre races peuvent apparaître; ainsi, LoNG et GREGORY (I974) ont trouvé que les fils des taureaux Angus avaient une croissance supérieure jusqu'au sevrage à celle des veaux issus de taureaux Hereford, contrairement à GAINEs et al. (I966) et à GREGORY et al. (I965). Ces divergences pourraient être aussi causées par des différences d'échantillonnage dûs à une grande variabilité entre taureaux intra races, ou être le fait de résultats obtenus à des époques différentes qui reflèteraient des orientations dans la sélection des races. Il faut également retenir le fait que ces expériences ont été réalisées dans d'autres pays, d'où des différences possibles de niveau génétique dans une même race par rapport aux animaux de cette expérience. Outre que les niveaux génétiques des races aient pu être différents en Argentine par rapport aux États-Unis d'Ämérique, des différences de conduite des animaux entre pays peuvent aussi avoir une incidence sur les expressions phénotypiques des différences entre races.

Dans notre étude, la faible valeur génétique additive maternelle de la race Hereford est compensée par une valeur génétique additive supérieure pour les effets directs; 1'inverse est observé pour la race Shorthorn. Il en résulte que les veaux des 2 races pures, ont des performances identiques notamment pour la croissance et le poids au sevrage (tab1. I). En fait, ces performances identiques sont obtenues différemment dans chacune des races. De ce fait il est donc probable que les conditions de pâturage des mères Shorthorn aient une incidence majeure sur la croissance de leur veau supérieure à celle des mères Hereford.

Quant aux niveaux d'hétérosis obtenus dans notre étude sur les effets directs, ils sont parmi les plus bas de ceux cités dans la littérature. Pour le poids à la naissance Mason (I966) a cité des valeurs estimées dans différents pays entre - I et +4 p. Ioo pour les Hereford et $A n g u s$, entre +2 et +7 p. Ioo pour les Hereford et Shorthorn, et entre + I et $+8 \mathrm{p}$. Ioo pour les Shorthorn et les Angus. Reprenant différents travaux, PRESTON \& WILLIS (I970) indiquent pour le même caractère que les valeurs d'hétérosis entre races britanniques pour différents travaux varient entre +5 et +6 p. IOO. LONG et GRÉGORY ont estimé 1'hétérosis entre les Hereford et Angus à +3 p. roo pour le poids de naissance. Pour la croissance absolue, nos résultats d'hétérosis qui varient entre $+\mathrm{I}$ et $+5 \mathrm{p}$. Ioo, se situent dans l'intervalle de variation (bien que parmi les plus faibles) des estimations d'hétérosis entre races britanniques qui va de o à +8 p. IOo (PRESTON \& WILLIS, I970; LONG \& GREGORY, I974; GREGORY et al., I965). Pour le poids au sevrage, la moyenne pondérée de I4 études différentes, calculée par CundifF (r970), est de 4,6 p. Ioo.

Nous avons obtenu peu de différences entre races pour les composantes génétiques additives directes et maternelles concernant la vitesse de croissance relative. Seule l'Angus semble posséder des composantes directes et maternelles légèrement supérieures. L'hétérosis sur les effets directs est quasiment nul. Ces résultats coïncident avec ceux de SмIтн et al. (I976); ces auteurs n'ont pas réussi à mettre en évidence des effets significatifs d'hétérosis et des différences significatives d'effets maternels entre ces trois races à viande britanniques, pour la croissance relative.

Ainsi sans considérer 1'efficacité de la production, pour obtenir la croissance absolue avant sevrage et les poids au sevrage les plus élevés, la meilleure combi- 
naison de races serait donc l'utilisation de taureaux Hereford sur vaches Shorthorn. Ainsi on pourrait bénéficier de la valeur génétique additive supérieure de la race Hereford pour les effets directs de celle supérieure de la race Shorthorn pour les effets maternels, et du maximum de vigueur hybride chez le veau ou d'hétérosis direct.

\section{2. - Comparaisons en croisement et en race pure de la race Charolaise avec les races britanniques}

Dans le tableau 3, nous avons comparé la race Charolaise à chacune des 3 races britanniques (contrastes I à 3) lorsqu'elles sont utilisées comme races paternelles de croisement sur des vaches de même type génétique. Ces différences entre race Charolaise et races britanniques représentent les différences de valeur génétique additive d'effets directs et les différences d'hétérosis individuel ou direct entre les croisés Charolais $\times$ races britanniques et races britanniques $\times$ races britanniques.

L'utilisation de la race Charolaise comme race paternelle de croisement a permis d'obtenir un poids de naissance, une croissance absolue et un poids au sevrage significativement supérieurs (sauf pour la croissance absolue par rapport à 1'Angus) à ceux de chacune des 3 races britanniques. Les travaux qui confirment cette supériorité de potentiel de croissance de la race Charolaise par rapport aux races britanniques, sont nombreux (par exemple: CundIFF, I970). De ce fait, la race Charolaise est une des races paternelles recommandées pour le croisement si l'objectif principal est d'accroître le poids des veaux au sevrage. De cette manière, il est possible de combiner les avantages de l'utilisation de vaches de petite taille avec ceux de la supériorité de croissance de la race Charolaise avec, en outre, l'avantage de vigueur hybride des veaux croisés. Cependant, la supériorité de poids des veaux à la naissance peut engendrer un accroissement de la fréquence des difficultés de naissance, surtout si aucun choix des taureaux n'a été fait pour limiter ces inconvénients. En croissance relative, les veaux issus de races britanniques sont supérieurs à ceux de race Charolaise (tab1. 3); il en résulte que l'avantage de potentiel de croissance de la race Charolaise sur les races britanniques est proportionnellement moins important au sevrage qu'à la naissance.

Dans le tableau 4, nous avons comparé les veaux de race Charolaise à ceux de chacune des races britanniques (contrastes I à 3) et à la moyenne de celles-ci (contraste 4). Ces différences représentent les différences de valeurs génétiques additives des effets directs et maternels. Comme en croisement, la Charolaise montre une supériorité de poids à la naissance, de croissance absolue et de poids au sevrage, avec une croissance relative plus faible. Toutes ces différences sont statistiquement significatives. Les différences sont proportionnellement plus élevées pour le poids à la naissance (de 24 à $42 \mathrm{p}$. IOO) que pour le poids au sevrage (de $\mathrm{I} 5$ à $\mathrm{I} 8 \mathrm{p}$. I oo), du fait de la meilleure croissance relative des races britanniques. Les différences (en p. Ioo) au sevrage entre les races Angus et Hereford avec la race Charolaise sont identiques à celles trouvées par SAGEBIEL et al. (I974), mais d'environ moitié de celles rapportées par PEACOCK et al. (I978) entre Angus et Charolais. Le meilleur niveau nutritionnel de l'expérimentation conduite par ces derniers auteurs, pourrait expliquer l'importance des différences rencontrées. Par rapport à SAGEBIEL et al. (I974) pour lesquels le niveau nutritionnel de l'expérience était certainement comparable à celui de l'essai des précédents auteurs, nous 


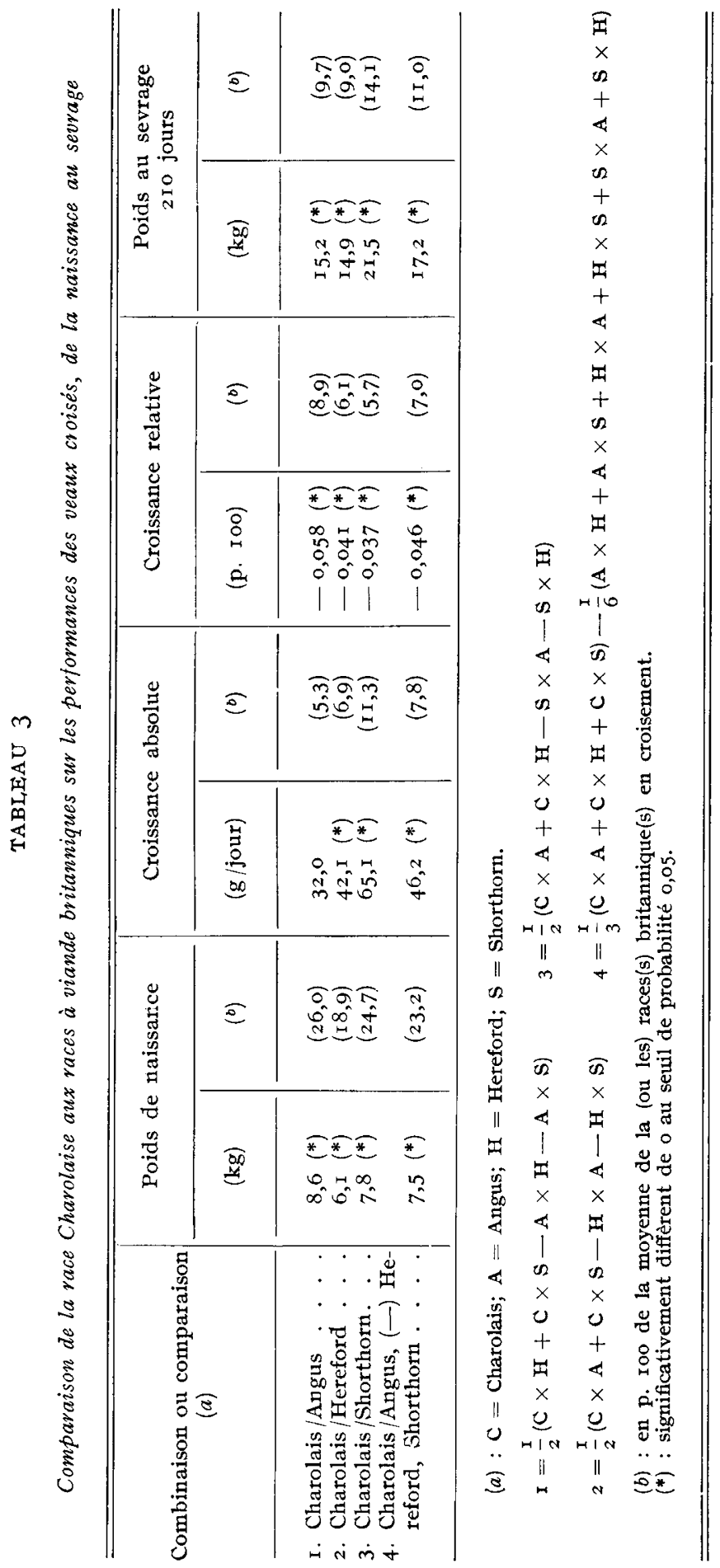




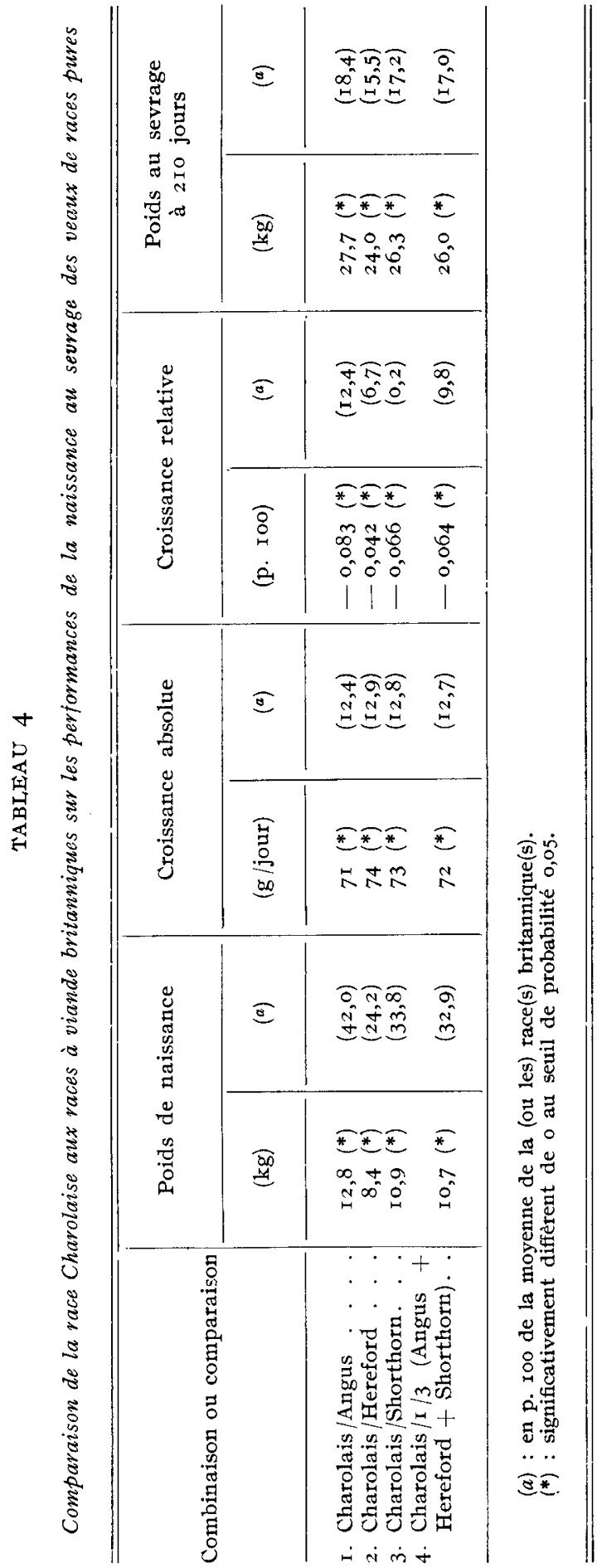


avons utilisé des reprođucteurs Charolais d'origine française qui probablement possèdent un potentiel de croissance supérieur à celui des taureaux Charolais nordaméricains utilisés par SAGEBIEL et al. (I974).

\section{Conclusions}

En ce qui concerne la croissance des veaux avant sevrage, les races britanniques sont assez comparables pour les différents critères analysés. La race Shorthorn possède un léger avantage par rapport aux deux autres races en ce qui concerne l'aptitude maternelle pour les poids à la naissance et au sevrage ainsi que pour la croissance absolue. L'Angus a montré des performances intermédiaires, sauf pour le poids à la naissance où elle est inférieure. La Hereford est plutôt supérieure pour les valeurs génétiques additives des effets directs de ces caractères de croissance. Pour la croissance relative, les différences sont encore plus faibles que pour les autres caractères; la race $A n g u s$ est celle qui a manifesté la meilleure valeur génétique additive pour les effets directs et maternels sur ce caractère.

Enfin, la race Charolaise surpasse les races britanniques tant en race pure qu'en croisement pour le poids de naissance et au sevrage, et la croissance absolue jusqu'au sevrage alors que sa croissance relative est inférieure à celle des races britanniques.

Reçu pour publication en novembre 1980.

\section{Remerciements}

Ce travail a été réalisé avec l'assistance technique de nombreuses personnes parmi lesquelles nous citerons plus particulièrement MM. R. SulLivan et R. Sofiak. Nous remercions également MM. F. MúNissier et I. SANTA Coloma pour leur collaboration à la traduction française et J. L. FOULIEY pour ses suggestions dans l'élaboration de ce mémoire.

\section{Summary}

Genetic components of Angus, Hereford, Shorthorn breeds and their Charolais crossbreds for preweaning growth traits.

Tests for hybrid vigour and differences between breed for individual (or transmitted) and maternal additive genetic components were done. The breeds used were: Aberdeen-Angus, Heveford, Shorthorn, and Charolais; In addition to purebreds, the crossbreds were all possible crosses among the British breeds and crosses of Charolais sires by British dams. The tests were done by building lineal combinations with the least squares breed and crossbred means for birth weight, preweaning absolute and relative daily gain and weaning weight adjusted to 2 Io days. The $45^{\circ}$ animals included in this study were born from 1960 to $x 965$ at the E.E.R.A. Balcarce (Argentina). Neither hybrid vigour nor differences among individual and maternal additive components were statistically significant.

Charolais crosses were 23 p. Ioo heavier at birth and II p. Ioo at weaning than the British breeds in crosses. Purebreds Charolais were $33 \mathrm{p}$. Ioo heavier at birth and $17 \mathrm{p}$. Ioo at weaning than British purebreds. 


\section{Références bibliographiques}

CUNDIFF L. V., I970. Experimental results on crossbreding for beef production. J. anim. Sci., 30, 694-705.

DEPAR'TAMENTO DE PRODUCTIÓN ANIMAL, I974. Cruzamientos con bovinos para carne. INTA, Est. Exp. A grop. Balcarce (Materiales Didacticos, Nros. 3).

DickERSON G., 1969. Experimental approaches in utilizing breed resources. anim. Breed. Abstr., 37, I9I-202.

FOULLEY J. L., LEFORT G., r978. Méthođes đ'estimation des effets directs et maternels en sélection animale. Ann. Génét. Sél. anim., 10, 475-496.

Gaines J. A., MC Clure W. H., Vogt D. W., Carter R. C., Kinaid C. M., r966. Heterosis from crosses among british breeds of beef cattle: fertility and calf performance to weaning. J. anim. Sci., 25, 5-I3.

Gregory K. E., Swiger I. A., Sumption I. J., Koch R. I., Rowden W. W., Ingalis J. L., I965. Heterosis in preweaning traits of beef cattle. J. anim. Sci., 24, 2 I-28.

HARVEY W. R., I964. Computing procedures for a generalized least-squares analysis program. U.S.D.A.A.R.S., Pres Analysis of variance conference, Colorado (mimeo).

KLOSTERMAN E. W., I972. Beef cattle size for maximum efficiency. J. anim. Sci., 34, 875-880.

LONG C. R., GREGORY K. E., I964. Heterosis and breed effects in preweaning traits of $A n g u s$, Heveford and reciprocal cross calves. J. anim. Sci., 39, II-I7.

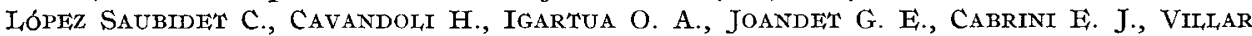
J. A., Sivor I. H., Hernandes O., Covas G., KUGLER W. F., 1963. Cruzas con Chavolais en la region pampeana. INTA, Est. Exp. Reg. Agrop. Balcavce, Bol. Tec., (6).

Mason I. I., I966. Hybrid vigour in beef Cattle. Anim. Breed. Abstr., 34, 453-473.

PEACOCK F. M., KOGER E. M., 1978. Weaning traits of Angus, Brahman, Charolais and $\mathrm{F}_{1}$ crosses of these breeds. J. anim. Sci., 47, 366-369.

PRESTON T. R., Willis M. B., I970. Intensive Beef Production. Pergamon Press Oxford, 544 p.

Sagebiel J. A., Krause Bob Sibbít G. F., Langford L., Dyer A. J., Lasley J. F., I974. Effect of heterosis and maternal influence an weaning traits on reciprocal crosses among $A n g u s$, Charolais and Heveford cattle. J. anim. Sci., 39, 47I-479.

Smith G. M., Fitzrugh Jr. H. A., Cundiff L. V., Cartwrigh'T T. C., Gregory K. E., I976. Heterosis for maturing patterns in Heveford, Angus and Shorthorn cattle. J. anim. Sci., 43, $380-388$.

TAYLOR St. C. S., I97I. The effect of body size on production efficiency in cattle. Ann. Génét. Sél. anim., 3, 85-90. 